\title{
Effects on blood pressure, cardiac mass and function after renal denervation in patients with resistant hypertension
}

\author{
Enver Tahir ${ }^{1 *}$, Lennart Well', Maxim Avanesov¹, Fabian J Brunner², Karsten Sydow¹, Gunnar Lund', Gerhard Adam', \\ Andreas Koops ${ }^{1}$
}

From 19th Annual SCMR Scientific Sessions

Los Angeles, CA, USA. 27-30 January 2016

\section{Background}

In recent years, catheter-based renal denervation (RDN) has been investigated as a promising strategy in the treatment of resistant hypertension. Due to a reduction of whole body sympathetic activity, additional benefits on cardiac adaptation have been proposed. The purpose of this study was to investigate the effect of RDN on blood pressure (BP) as well as cardiac mass and function via cardiac magnetic resonance imaging (CMRI).

\section{Methods}

RDN was performed on 15 patients with a history of resistant hypertension ( 9 male and 6 female, mean age 67.2 years). Office and ambulatory long term blood pressures were measured before and 12 months after RDN. For quantitative CMRI, an electrocardiographically triggered steady-state free precession (SSFP) cine sequence (TR/TE, $3.2 / 1.6 \mathrm{~ms}$; pixel-size, $1.7 \mathrm{~mm} \times 1.7 \mathrm{~mm}$ ) was performed in short- and long-axis views before and 12 months after RDN. Quantitative analysis included end-diastolic (EDV) and end-systolic volumes (ESV), stroke volume (SV), left ventricular ejection-fraction (EF) as well as end-diastolic (EDMM) and end-systolic myocardial mass (ESMM). CMRI data were analyzed by two independent observers using the HeAT-Software. Data are given as the mean of both observers. Statistical analysis was performed using GraphPad Prism 4 and Excel, Microsoft.

\section{Results}

In patients with resistant hypertension, RDN let to a significant decrease of EDMM $(162.8 \pm 52.4 \mathrm{~g}$ vs $152.5 \pm 52.0 \mathrm{~g}$;

'Diagnostic and Interventional Radiology, University Hospital Eppendorf, Hamburg, Germany

Full list of author information is available at the end of the article $\mathrm{p}<0.05)$ and ESMM $(166.5 \pm 54.5 \mathrm{~g}$ vs $154.9 \pm 53.23 \mathrm{~g}$; $\mathrm{p}<0.05)$ within 12 months after intervention. EDV (163.0 \pm $38.6 \mathrm{ml}$ vs $168.1 \pm 49.8 \mathrm{ml})$, ESV $(70.4 \pm 33.0 \mathrm{ml}$ vs $72.0 \pm$ $39.8 \mathrm{ml})$, SV $(92.7 \pm 20.7 \mathrm{ml}$ vs $96.1 \pm 30.6 \mathrm{ml})$ and $\mathrm{EF}$ $(0.583 \pm 0.116 \%$ vs $0.588 \pm 0.126 \%)$ did not change on a significant level. BP measurements revealed a significant decrease of the minimal diastolic BP in ambulatory long term measurements $(53.3 \pm 9.2 \mathrm{mmHg}$ vs $48.8 \pm 12.8$ $\mathrm{mmHg} ; \mathrm{p}<0.05)$. No additional significant changes in average, systolic or diastolic, office or ambulatory, diurnal or nocturnal BP measurements was detected.

\section{Conclusions}

RDN lead to a decrease of cardiac mass within 12 months after intervention without a significant change of left ventricular function. Additionally, minimal diastolic BP showed a significant decrease in ambulatory measurements, whereas no other significant changes in BP levels were detected. Despite a rather small effect on blood pressure, additional benefits of RDN regarding cardiac adaptation can be observed. Further investigations might lead to a specified selection of patients who benefit from $\mathrm{RDN}$ beyond reduction of $\mathrm{BP}$.

\section{Authors' details \\ 'Diagnostic and Interventional Radiology, University Hospital Eppendorf, Hamburg, Germany. ${ }^{2}$ General and Interventional Cardiology, University Heart} Center Hamburg, Hamburg, Germany.

Published: 27 January 2016

doi:10.1186/1532-429X-18-S1-P106

Cite this article as: Tahir et al:: Effects on blood pressure, cardiac mass and function after renal denervation in patients with resistant hypertension. Journal of Cardiovascular Magnetic Resonance 2016 18(Suppl 1):P106 